Note

\title{
Research, education and clinical practice: an example of a learning community in the field of Speech and Language Therapy
}

Eline Alons ${ }^{1}$, Manon van Daatselaar ${ }^{1}$, Dorien Preuter ${ }^{1}$, Kim van der Zalm¹ ${ }^{1}$ Lizet van Ewijk $^{1}{ }^{*}$

1. Speech and Language Therapy, Institute for Allied Health Professionals, HU University of Applied Sciences Utrecht, The Netherlands

*Corresponding author: lizet.vanewijk@hu.nl

Received: 30 January 2017; Accepted: 23 February 2017; Published: 12 May 2017

Keywords: applied research; aphasia; student involvement

\section{Introduction}

The HU University of Applied Sciences Utrecht in The Netherlands has an honours program based on a star-system, in which students have the opportunity to earn one or more stars on top of their BA curriculum, which helps them to distinguish themselves from other students. There are five areas in which students can extend their knowledge; expertise, innovation \& dissemination, international orientation, professional drive and leadership. In addition to the extra work students do, they write a reflection report on their experiences. Both are then evaluated by an honours committee. If you receive a positive review, you will be awarded a Star Certificate. In this particular project, four Speech and Language Therapy (SLT)-students participated in an innovative research project involving the development of a new diagnostic tool for clinical practice. The project provides a great example of how innovation takes place, when research, education and clinical practice meet. The students shared their experiences at the Honours Conference 2016 in Utrecht. This note is a summary of their presentation.

\section{Contents of the honours program}

To understand the contents of the honours program the students participated in, a little background information about speech and language therapy is needed.

A Speech and Language Therapist (SLT) is specialized in communication disorders. Communication disorders can be caused by multiple reasons. Aphasia is a communication disorder, caused by acquired brain damage. People with aphasia may have problems in speaking, reading, writing and language comprehension. Many people with aphasia have problems in finding words and naming objects, which may affect their daily communication. Words are stored in our brain, in the so-called lexicon. Several factors affect the speed and accuracy of word retrieval. Two of these factors are frequency of occurrence of a word and 
age of acquisition. The influence of frequency on word retrieval is commonly described; the more often the word is used, the easier and faster we retrieve the word from our brain. Recent research has shown that also the age of acquisition is an important variable in the organisation of the words in the brain. The younger the word is acquired, the faster and more accurately they are retrieved, and the better they seem to be preserved in our brain after damage.

Word finding difficulties can be diagnosed by a test. The most common test SLTs use is the Boston Naming Test (BNT, see Kaplan, Goodglass \& Weintraub, 1983). This instrument is outdated and it is not standardized for Dutch. Furthermore, the diagnosis word finding difficulties by the BNT is only based on the frequency factor. Therefore, the Research group Speech Language Therapy at HU University of Applied Sciences is developing a new test. It is based on the most recent insights in cognitive linguistics and includes both frequency and age of acquisition factors.

This is where the honours program began. The students were involved in selecting items for the test and helped to set up and perform a survey to determine the age of acquisition of these words.

To create the naming test, words had to be collected for six categories (table 1 ).

Table 1. The six categories for the items of the new naming test

Early age of acquisition and high frequency Late AoA and high frequency (e.g. taxi) (e.g. house)

Early age of acquisition and middle frequency Late AoA and middle frequency

Early age of acquisition and low frequency Late AoA and low frequency (e.g. iron) (e.g. diaper)

The students searched for words to fill these categories. Frequency of selected words was easy to find in a database (SUBTLEX-NL, see Keuleers, Brysbaert \& New, 2010), but there is little data available about the age of acquisition of Dutch words. Research was necessary to determine the exact age of acquisition (AoA) for the words that will be included in the test. Earlier research has shown that AoA can be determined by asking people at what age they think they acquired a specific word. Therefore, the students performed a survey to determine the age of acquisition of the selected words. Almost 100 participants estimated their AoA for the words in the survey. Based on these data, and the frequency of items, a final selection was made for the test.

The naming test will probably be published on paper and online. At the moment there are no other tests for speech and language therapy online, so this is an innovative concept in the discipline. Collaboration with ICT-students from the faculty Nature and Technology was started to explore the possible application of the test in digital form. The collaboration was interactive, because the ICT-students also needed help in developing an application for people with aphasia. The students informed the ICT-students in order to make the app appropriate for people with aphasia.

\section{Learning experiences}

By participating in this research project, experience was gained in the interaction between doing research and the clinical practice during education. Collaboration between 
researchers, clinical practitioners and students creates innovation. In this research, principles of Evidence Based Practice (EBP, relating to the best available research evidence, clinical expertise and the client preferences) are combined to create an evidence based naming task.

Through the collaboration with the faculty Nature and Technology, the students learned to talk comprehensibly about aphasia with a layperson. Furthermore, they experienced the possibilities of innovative technology, which is little used by SLTs. There is a lot of potential for SLTs for including more technology in their therapy.

Overall, knowledge is expanded and deepened in different ways by participating in this honours project. Knowledge is gained about doing research, which is part of evidence-based practice. Developing a test takes many years, money and patience. Besides, the students learned more about the steps that needed to be made in this kind of research. Moreover, more specific knowledge about word finding difficulties is gained.

\section{References}

Kaplan, E.F., Goodglass, H. \& Weintraub, S. (1983). The Boston Naming test (2nd edition). Philadelphia: Lea \& Febiger.

Keuleers, E., Brysbaert, M. \& New, B. (2010). SUBTLEX-NL: A new frequency measure for Dutch words based on film subtitles. Behavior Research Methods, 42(3), 643-650. 